\title{
Accuracy of calibrated data from the SDSS moving object catalog, absolute magnitudes, and probable lightcurves for several asteroids
}

\author{
A. Galád ${ }^{1,2}$ \\ 1 Dept. of Astronomy, Physics of the Earth, and Meteorology, FMFI, Comenius University, 84248 Bratislava, Slovakia \\ 2 Astronomical Institute, AS CR, 25165 Ondřejov, Czech Republic \\ e-mail: galad@fmph.uniba.sk
}

Received 8 January 2010 / Accepted 8 February 2010

ABSTRACT

\begin{abstract}
It would seem that the calibrated observations obtained by the Sloan Digital Sky Survey (SDSS) by themselves would be, at best, marginally useful for the secure determination of asteroid rotation lightcurves, mostly due to the scarcity of data for a particular object in a given apparition as well as because of the sometimes low photometric quality of the SDSS data. Despite these shortcomings, it was decided to see if the SDSS data could be used to help find the lightcurve parameters of at least some asteroids. Observations of ten asteroids obtained by the SDSS are compared here with lightcurves obtained by asteroid photometric stations using dense data sets. Three asteroids observed during the same apparition as the SDSS observations served to determine the accuracy of the SDSS data. Except for occasional outliers identified on the basis of deviating color indices, the accuracy of the observations was found to be about 0.03 mag in the $V$ band on average, which is a generally accepted level of quality for most asteroid photometry.

In addition to the ten asteroids with known lightcurves, another 54 asteroids without known lightcurves, but with more than 20 observations by SDSS, were also examined to derive their absolute magnitudes $(H)$ and plausible composite lightcurves. Lightcurve analyses of (12104) Chesley, (32257) $2000 \mathrm{OW}_{52}$, (39132) $2000 \mathrm{WU}_{58}$, (156751) $2002 \mathrm{XL}_{92}$, (219686) $2001 \mathrm{WE}_{37}, 1992 \mathrm{WW}_{6}$, and $2007 \mathrm{EP}_{39}$ are presented. The asteroids studied in this paper were found to be mostly fainter than predicted from the $H$ values given by the Minor Planet Center in its Orbit Database. The difference between the $H$ values slightly correlates with the lightcurve amplitude.
\end{abstract}

Key words. minor planets, asteroids: general - techniques: photometric

\section{Introduction}

Photometry is still the most widely used method to derive rotation properties and shapes of asteroids. Despite some of the advantages of this method, such as being technically easy and the tremendous number of available targets, even for small sized telescopes, there are also some drawbacks. These include the frequent requirement for long and preferably continuous runs, the difficulty in forming collaborations when observations at different longitudes are needed because of ambiguous results or complex lightcurves, and - most important - the strong bias towards objects that are bright, fast rotating, and/or have simple lightcurves with large amplitudes.

The Sloan Digital Sky Survey (SDSS) provides astrometric and photometric observations (color indices, in particular) of many objects including asteroids (Ivezić et al. 2002) using the $2.5 \mathrm{~m}$ telescope located at Apache Point Observatory in New Mexico (York et al. 2000). The fourth release of the SDSS Moving Object Catalog (MOC) (Jurić et al. 2007) contains 471569 observations. Of those, 220101 were matched to 104449 known objects, meaning that each asteroid in the catalog was observed, on average, a little more than twice. Since the SDSS observations may span more than one apparition, they would not in general be expected to be useful for finding asteroid rotation periods. However, some asteroids were observed more often than others, and so an examination of the SDSS MOC was made to see to what extent these observations could complement the database of currently known lightcurves.

\section{Sampling and accuracy}

The first step in this examination was to extract all observations for individual asteroids. Those with very large errors in color indices, $>0.5 \mathrm{mag}$, probably due to contamination with background objects or nonphotometric conditions, were flagged. Surprisingly, a few asteroids were found to have been observed more than 20 times and mostly within one apparition, between 2005 September 3 and December 1 (Table 1). Since the time span covered several weeks, only simple lightcurves with large amplitudes could, in principle, be resolved unambiguously, but only if all the observations were of high quality. This was not always the case. While analyzing SDSS color indices, Fukugita et al. (1996) showed that the accuracy of observations for given stars (and dwarfs) was $\approx 0.04$ mag in the $V$ band. For comparison, many asteroid photometric stations can produce high quality data with errors below 0.03 mag for bright objects. On the other hand, these data are often only relative, not calibrated.

The transformation of the SDSS observations to Johnson $V$ band is given by

$V=r+0.44 \times(g-r)-0.02$

where $g$ and $r$ are the magnitudes in the SDSS bands (Juric et al. 2002). Therefore, formal errors of individual observations could also be estimated accordingly. These errors were usually found to be within $0.03 \mathrm{mag}$, but occasionally they exceeded that limit. Worse yet, actual errors could be even larger. Based on this 
Table 1. Asteroids observed more than 20 times.

\begin{tabular}{rlllllllll}
\hline \hline Asteroid & $N$ & Asteroid & $N$ & Asteroid & $N$ & Asteroid & $N$ & Asteroid & $N$ \\
\hline$(1947)$ & 25 & $(63376)$ & 23 & $(119651)$ & 21 & $(153879)$ & $22(21)$ & $2005 \mathrm{RF}_{47}$ & 21 \\
$(8914)$ & 24 & $(65200)$ & 23 & $(124135)$ & $28(26)$ & $(153937)$ & 22 & $2005 \mathrm{SF}_{286}$ & 21 \\
$(12104)$ & 24 & $(68480)$ & 23 & $(125136)$ & $22(21)$ & $(156751)$ & 22 & $2005 \mathrm{SS}_{287}$ & 21 \\
$(32257)$ & $21(20)$ & $(79530)$ & 22 & $(125779)$ & 24 & $(160318)$ & $22(21)$ & $2005 \mathrm{UV}_{108}$ & $21(19)$ \\
$(35774)$ & $21(20)$ & $(88005)$ & 22 & $(128018)$ & 22 & $(214479)$ & 22 & $2005 \mathrm{UC}_{130}$ & 23 \\
$(39132)$ & 23 & $(89891)$ & 21 & $(128343)$ & $24(23)$ & $(215187)$ & $23(21)$ & $2005 \mathrm{UO}_{213}$ & 23 \\
$(42306)$ & $24(22)$ & $(101812)$ & 21 & $(148844)$ & $21(20)$ & $(219686)$ & $21(20)$ & $2005 \mathrm{UU}_{396}$ & 23 \\
$(57252)$ & $21(19)$ & $(106590)$ & 23 & $(149182)$ & 25 & $(221267)$ & 21 & $2005 \mathrm{VR}_{131}$ & $24(23)$ \\
$(58673)$ & $22(21)$ & $(110634)$ & 22 & $(151057)$ & 21 & $(225873)$ & 21 & $2005 \mathrm{VS}_{131}$ & 22 \\
$(62781)$ & 21 & $(110755)$ & $24(23)$ & $(153695)$ & $21(20)$ & $1992 \mathrm{WW}_{6}$ & 23 & $2007 \mathrm{EP}_{39}$ & 23 \\
$(63029)$ & 22 & $(119574)$ & 23 & $(153822)$ & 22 & $2003 \mathrm{GG}_{51}$ & $22(19)$ & & \\
\hline
\end{tabular}

Notes. The number of observations within a single apparition is in parenthesis.

Table 2. The most frequently observed asteroids by SDSS that have known lightcurves from the independent study elsewhere.

\begin{tabular}{|c|c|c|c|c|c|}
\hline \multicolumn{2}{|c|}{ Asteroid } & $U$ & $\mathrm{LC}$ & $N$ & Analysed \\
\hline$(728)$ & Leonisis & 3 & 2007 & $17(15)$ & yes \\
\hline (1534) & Näsi & $3-$ & 2007 & $15(12)$ & yes \\
\hline (1829) & Dawson & 2 & 2002 & & \\
\hline (1829) & Dawson & 3 & 2005 & $8(7)$ & yes \\
\hline (1829) & Dawson & 3 & 2007 & & \\
\hline (2453) & Wabash & 3 & 2005 & $14(11)$ & yes \\
\hline (2659) & Millis & 3 & 1991 & $11(10)$ & yes \\
\hline (2903) & Zhuhai & 2 & 2000 & $10(8)$ & no \\
\hline (4467) & Kaidanovskij & 1 & 2005 & $14(13)$ & no \\
\hline (5288) & Nankichi & 2 & 2007 & $10(9)$ & no \\
\hline (5448) & Siebold & 3 & 2007 & $10(9)$ & no \\
\hline (7760) & $1990 \mathrm{RW}_{3}$ & 3 & 2005 & $8(8)$ & yes \\
\hline (8405) & Asbolus & 2 & 1996 & & \\
\hline (8405) & Asbolus & 3 & 1997 & & \\
\hline (8405) & Asbolus & 3 & 2005 & $14(12)$ & yes \\
\hline (9368) & Esashi & 3 & 2007 & $10(10)$ & yes \\
\hline (16706) & Svojsík & 1 & 2003 & $10(9)$ & no \\
\hline (30825) & $1990 \mathrm{TG}_{1}$ & 3 & 2005 & $10(9)$ & yes \\
\hline (35690) & $1999 \mathrm{CT}_{21}$ & 2 & 2000 & $14(14)$ & yes \\
\hline
\end{tabular}

Notes. $U$ is the index of quality for rotation period determination, LC denotes the year at which the best lightcurve was obtained, $N$ is the number of (reliable) SDSS observations in 2005.

analysis, it did not seem likely that anything better than ambiguous rotation periods could be found using the SDSS data.

\section{Comparison with known lightcurves}

The best way to estimate the quality of SDSS observations was to compare them with high-quality photometric observations, preferably obtained at the same apparition. None of the asteroids most frequently observed by SDSS listed in Table 1 was found to have known lightcurve parameters in the asteroid lightcurve database (LCDB, Warner et al. 2009). In order to proceed further, the limit for the number of SDSS observations of a particular object had to be decreased. Table 2 lists asteroids observed at least ten times by SDSS that were studied by other observers. Only eight SDSS observations were required in those cases where other lightcurve observations were obtained during the same apparition. For each asteroid, only the best results (period and amplitude) were used to compare with the SDSS data. This was determined by the $U$ quality code assigned in the LCDB, which is $U=3$ for secure and unambiguous results,
$U=2$ when the results are reasonably secure (still considered to be sufficient for statistical studies) and $U=1$ when the results are tentative and may be incorrect.

It was quickly recognized that several SDSS observations (for example, faint outliers) with even low formal errors were probably erroneous and had to be excluded from further analysis. Usually, these observations were also apparent outliers in terms of color indices, like $(B-V),(i-z)$, and others.

After the SDSS data were light-time corrected and period analysis was done with the Fourier series method (Harris et al. 1989), composite lightcurves were constructed for ten objects from Table 2. Table 3 lists the absolute magnitude $H$, the slope parameter $G$, synodic rotation period $P$, the estimated period error $\sigma_{P}$, the maximum synodic-sidereal period difference $\Delta P$ for the period covered by observations (Pravec et al. 2005), and the amplitude of the lightcurve $A$ according to a Fourier fit of order $n$ for each asteroid. A high-order Fourier fit is usually not recommended in cases of limited data. Sometimes even a third-order fit would be too much. The slope parameter was often assumed to be $G=0.15$ (enclosed by parentheses in Table 3 ), although in some cases a different value could be found so that the root mean square residuals of the fit to the SDSS data $\mathrm{RMS}_{\mathrm{SDSS}}$ was lower. The main reason for resorting to the default value was the low degree of freedom.

In Figs. 1 through 10, the $y$-axis, $V(0)$, is the reduced $V$ magnitude at zero solar phase angle (its mean value is the absolute magnitude $H$ ). Composite lightcurves of high quality were constructed for three asteroids. In these cases, relative lightcurve data from other photometric stations could be joined with data from SDSS because they were obtained at the same apparition. Only simple composite lightcurves could be constructed for the other asteroids because a lower-order Fourier fit had to be used.

(728) Leonisis. Lightcurve observations from two nights in 2007 by Roy ${ }^{1}$ revealed $P=5.586 \pm 0.006 \mathrm{~h}$ and $A=0.19 \mathrm{mag}$. An analysis of 15 out of 17 SDSS observations that were obtained in 2005 (two points were outliers based on their deviating color indices) shows that the data fit very well to a few discrete rotation periods, like $3.6358 \mathrm{~h}$, or $4.5253 \mathrm{~h}$, as well as the true synodic rotation period (Fig. 1). Using the SDSS observations allowed the period to be found more precisely. The best fit is for $G=0.15$. Its error seems to be small in this case, but it is not quoted for any asteroid due to the small number of SDSS observations.

\footnotetext{
1 See the lightcurve on R. Behrend's web page available at http:// obswww. unige.ch/ behrend/page_cou.html
} 
Table 3. Results of analysis for ten asteroids from Table 2.

\begin{tabular}{|c|c|c|c|c|c|c|c|c|c|c|c|}
\hline Asteroid & & $H_{\mathrm{MPC}}$ & $H$ & $G$ & $\begin{array}{l}P \\
\mathrm{~h}\end{array}$ & $\begin{array}{l}\sigma_{P} \\
\mathrm{~h}\end{array}$ & $\begin{array}{l}\Delta P \\
\mathrm{~h}\end{array}$ & $\begin{array}{c}A \\
\text { mag }\end{array}$ & $n$ & $\begin{array}{c}\text { RMS } \\
\text { mag }\end{array}$ & $\begin{array}{c}\mathrm{RMS}_{\mathrm{SDSS}} \\
\mathrm{mag}\end{array}$ \\
\hline (728) & Leonisis & 12.8 & 13.04 & 0.15 & 5.5789 & 0.0004 & 0.0002 & 0.33 & 3 & & 0.015 \\
\hline (1534) & Näsi & 11.7 & 11.96 & 0.19 & 7.9338 & 0.0003 & 0.0005 & 0.51 & 3 & & 0.011 \\
\hline (1829) & Dawson & 12.5 & 12.71 & $(0.15)$ & 4.25009 & 0.00008 & 0.00026 & 0.28 & 6 & 0.017 & 0.029 \\
\hline (2453) & Wabash & 11.2 & 11.39 & $(0.15)$ & 6.8792 & 0.0001 & 0.0003 & 0.63 & 5 & 0.029 & 0.023 \\
\hline (2659) & Millis & 11.2 & 11.59 & -0.08 & 6.1242 & 0.0008 & $<0.0001$ & 0.84 & 2 & & 0.035 \\
\hline (7760) & $1990 \mathrm{RW}_{3}$ & 13.2 & 13.61 & $(0.15)$ & 25.911 & 0.003 & 0.010 & 0.35 & 6 & 0.020 & 0.037 \\
\hline (8405) & Asbolus & 9.0 & 9.26 & 0.35 & 4.4682 & 0.0003 & $<0.0001$ & 0.34 & 1 & & 0.023 \\
\hline (9368) & Esashi & 13.3 & 13.71 & 0.15 & 2.9196 & 0.0002 & 0.0001 & 0.14 & 2 & & 0.015 \\
\hline$(30825)$ & $1990 \mathrm{TG}_{1}$ & 14.8 & 14.81 & $(0.15)$ & $(2.6243)$ & & 0.0001 & 0.11 & 2 & & 0.022 \\
\hline$(35690)$ & $1999 \mathrm{CT}_{21}$ & 14.0 & 14.47 & $(0.15)$ & 9.028 & 0.003 & $<0.001$ & 0.50 & 2 & & 0.061 \\
\hline
\end{tabular}

Notes. $H_{\mathrm{MPC}}$ is the absolute magnitude used in Minor Planet Center. Values in parenthesis are assumed. RMS is related to all available observations (including SDSS).

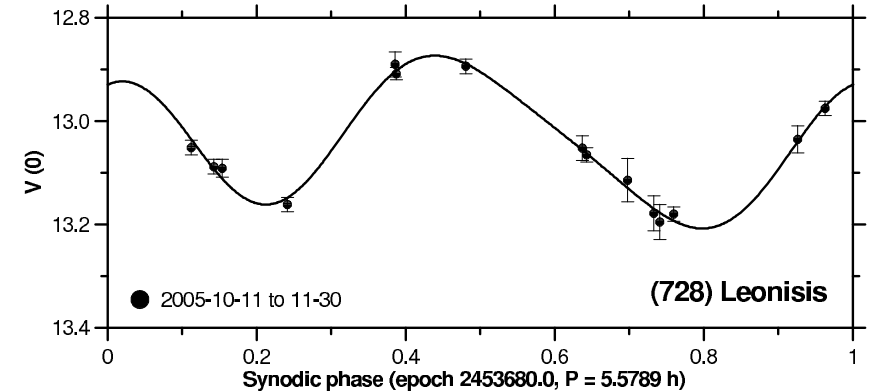

Fig. 1. Composite lightcurve f (728) Leonisis.

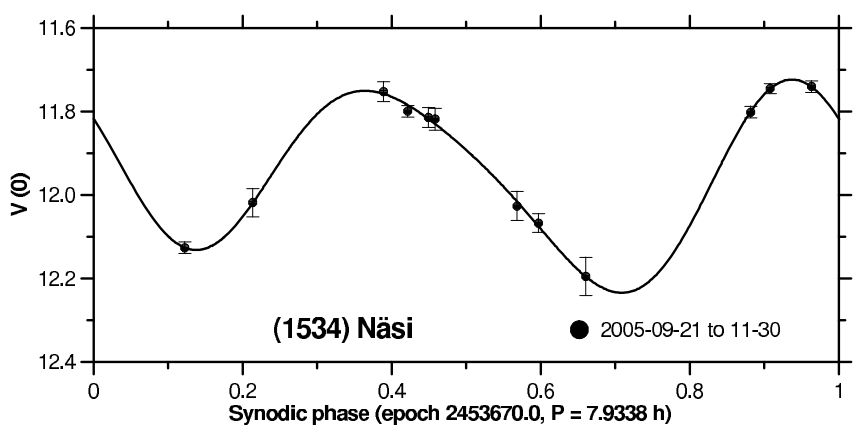

Fig. 2. Composite lightcurve of (1534) Näsi.

(1534) Näsi. Based on three sessions, Sauppe et al. (2007) estimated $P=7.94 \pm 0.02 \mathrm{~h}$ and $A \approx 0.35 \mathrm{mag}$. Their result was confirmed using SDSS observations after two apparent outliers were excluded for their color indices. However, the new result, $P=7.929 \mathrm{~h}$, was still questionable due to large residuals $\mathrm{RMS}_{\text {SDSS }}(\approx 0.09 \mathrm{mag})$. Careful inspection of color indices revealed that one more data point was an outlier, though just slightly. After it was excluded from the solution, $\mathrm{RMS}_{\mathrm{SDSS}}$ was greatly reduced (Fig. 2, Table 3).

(1829) Dawson. Lightcurves for this asteroid were available from three apparitions. Stephens (2003) obtained $P=4.243 \pm$ $0.003 \mathrm{~h}$ and $A=0.09 \mathrm{mag}$ in 2002; Pray (2006) obtained $P=4.254 \pm 0.001 \mathrm{~h}$ and $A=0.28 \mathrm{mag}$ in 2005; and Higgins ${ }^{2}$ obtained $P=4.2463 \pm 0.0009 \mathrm{~h}$ and $A=0.20 \mathrm{mag}$ in 2007 . One outlying SDSS observation was excluded from analysis while the remaining seven were joined to 110 observations in

\footnotetext{
2 http://www.david-higgins. com/Astronomy/asteroid/ lightcurves.htm
}

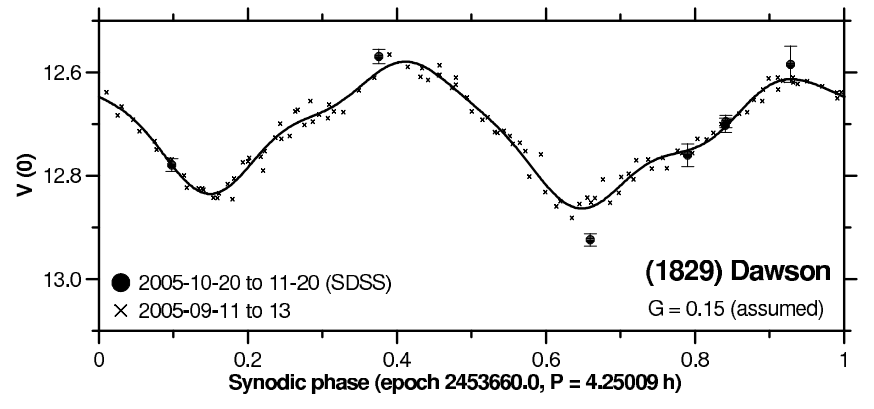

Fig. 3. Composite lightcurve of (1829) Dawson. Seven high-quality SDSS observations were joined to those by Pray (2006) (two SDSS observations are too close to each other).

three sessions by Pray (Fig. 3), which improved the synodic period solution. If the shape of the lightcurve remained the same during the whole period of observations, the accuracy of SDSS observations, determined by the RMS $\mathrm{SDSS}_{\text {, would be }}$ $\approx 0.03 \mathrm{mag}$ (Table 3 ). In fact, the accuracy could be better since the lightcurve probably changed at the larger solar phase angles covered by SDSS observations (the observations indicate a larger amplitude). Due to the small number of SDSS observations, the default of $G=0.15$ is used in Table 3. Nominally, the best result is when $G=0.11$, which would lead to $H=12.65$. The other parameters would remain the same.

(2453) Wabash. The SDSS observations were obtained at the same apparition as by Pray (2006), who recorded 163 observations during four sessions. He derived $P=6.878 \pm 0.001 \mathrm{~h}$ and $A \approx 0.67$ mag. Initially, only one out of 14 SDSS observations was excluded. However, all periods that matched the SDSS observations differed from the rotation period found by Pray. By removing another SDSS observation, a slight outlier in terms of color indices, it was possible to match the accepted period. Yet another SDSS observation was removed for the same reason. Based on the 11 remaining SDSS observations and assuming $G=0.15$, the final result was $P=6.8798 \pm 0.0006 \mathrm{~h}$ when using a second-order Fourier fit $\left(\mathrm{RMS}_{\mathrm{SDSS}}=0.044 \mathrm{mag}\right)$. With a third-order fit, $P=6.8793 \pm 0.0006 \mathrm{~h}\left(\mathrm{RMS}_{\mathrm{SDSS}}=0.031 \mathrm{mag}\right)$. In both cases, $A$ was $\approx 0.8$ mag.

Since the original relative lightcurve data by Pray were available, they were joined with the SDSS observations. The first step was to shift Pray's relative data to minimize the errors in the Fourier fit. This resulted in values for $P$ and $A$ that were just 


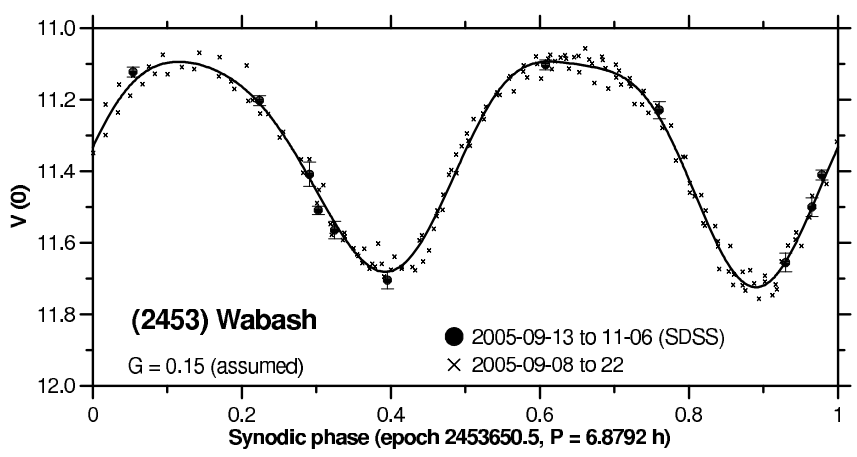

Fig. 4. Composite lightcurve of (2453) Wabash. Eleven high-quality SDSS observations were joined to those by Pray (2006).

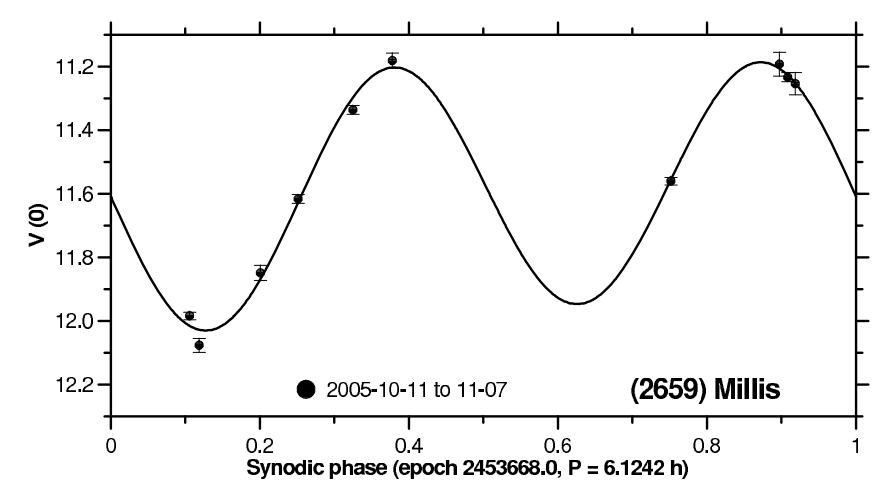

Fig. 5. Composite lightcurve of (2659) Millis.

slightly different from the published values. Then the SDSS data were joined in, which resulted in no significant change in the period or amplitude (Fig. 4). The quality of 11 SDSS observa-

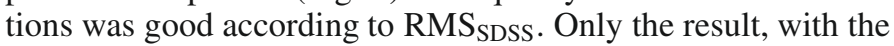
default $G$ value is shown in Table 3. The best result is when $G=0.10$, but all parameters (except for $H$, which would change to 11.34 ) would be the same. It is interesting that the largest error bar (formal errors) of individual SDSS observations (including those three which were excluded) was 0.034 mag.

(2659) Millis. Wisniewski et al. (1997) derived $P=6.132 \pm$ $0.002 \mathrm{~h}, A=0.53 \mathrm{mag}$ as well as $H=11.65 \pm 0.02$ (assuming $G=0.09$ ) for this asteroid. Several plausible periods were found using 10 out of 11 SDSS observations, with one of the periods close to the published value. The amplitude, of the lightcurve, however, was larger (Fig. 5). Only a second-order Fourier fit could be used. With $G=0.15$, this gave $\mathrm{RMS}_{\mathrm{SDSS}}=0.044 \mathrm{mag}$ and $H=11.82$. The best result was achieved with a negative value for $G$ (Table 3 ). The values for $H$ derived in this work and by Wisniewski et al. differ significantly from the value currently used by the Minor Planet Center (MPC), i.e., $H_{\mathrm{MPC}}=11.2$.

(7760) $1990 \mathrm{RW}_{3}$. Pray et al. (2006) reported 11 sessions of relative data with 284 points obtained between 2005 October 2 and November 1 . Those data were reanalyzed here, minimizing the residuals to get the best fit. This produced slightly different values for the period and amplitude $(25.902 \mathrm{~h}$ and $0.35 \mathrm{mag}$ instead of Pray's $25.940 \mathrm{~h}$ and $0.32 \mathrm{mag}$ ). The SDSS provided just eight additional calibrated observations of high quality (i.e., with low formal errors) in the period of 2005 October 20 and November 19. Their inclusion into the lightcurve resulted in practically no change to the shape of the lightcurve, although at

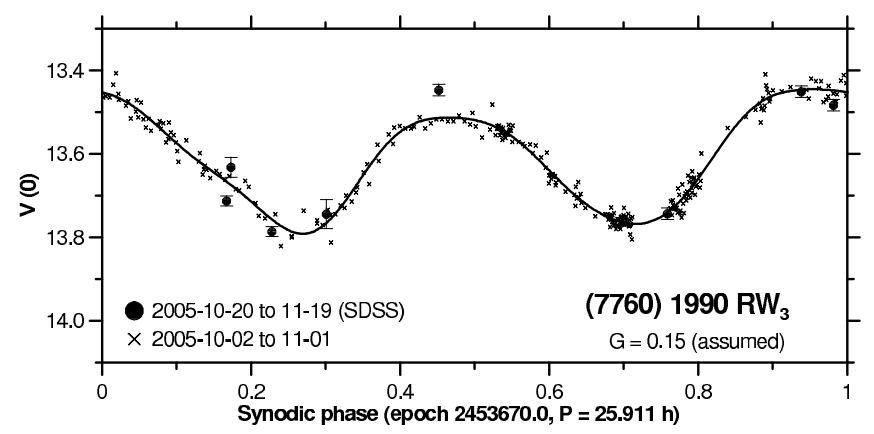

Fig. 6. Composite lightcurve of (7760) $1990 \mathrm{RW}_{3}$. All eight SDSS observations were joined to those by Pray et al. (2006).

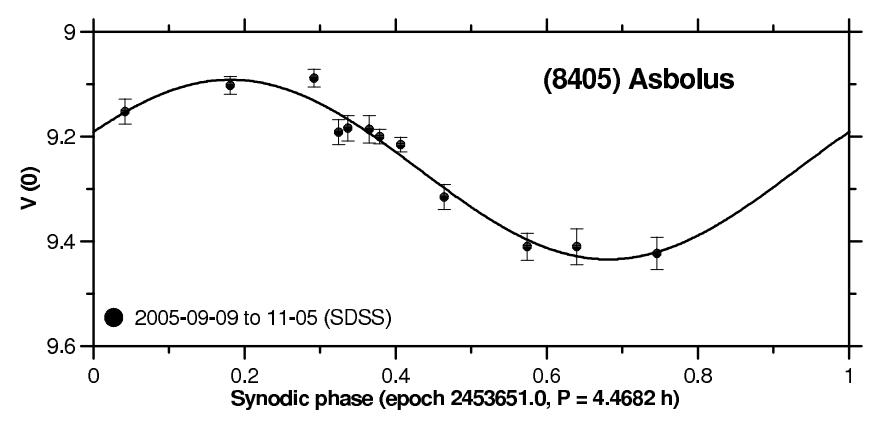

Fig. 7. Composite lightcurve of (8405) Asbolus.

least one SDSS observation deviated from the lightcurve more than expected.

The value for $G$ could not be precisely determined from the small number of observations. When using $G=0.15$, $P=25.911 \pm 0.003 \mathrm{~h}, A=0.35 \mathrm{mag}$ and $H=13.61$ (Fig. 6). The difference between synodic and sidereal rotation periods $\Delta P$ could be, at most, $0.010 \mathrm{~h}$ over the entire observational interval. Therefore it cannot explain the larger deviations of individual calibrated observations from the mean lightcurve allowed by the formal errors. The object was bright $(V<17)$, but the $\mathrm{RMS}_{\mathrm{SDSS}}$ of all 8 SDSS observations was 0.037 mag on average, while the formal errors (error bars) of individual observations were lower. The MPC value of $H_{\mathrm{MPC}}=13.2$ is not consistent with the SDSS data.

(8405) Asbolus. The rotation period of this Centaur, based on a bimodal lightcurve, is approximately $8.9 \mathrm{~h}$ (Brown \& Luu 1997, Davies et al. 1998; Brucker et al. 2008), while the period based on spectroscopic changes (albedo variation) is onehalf that value, or about $4.47 \mathrm{~h}$ (Kern et al. 2000). A monomodal lightcurve was assumed for this work, meaning the shorter period. Two SDSS data points, one an obvious outlier and the other a marginal one, were excluded to construct the lightcurve (Fig. 7). The lowest $\mathrm{RMS}_{\text {SDSS }}$ was obtained for a high value of $G$ (Table 3), but its true value may be different, because all observations were at low phase angles $\left(0.2^{\circ}-4.4^{\circ}\right)$. On the other hand, the value for $H$ had a low error. The reported amplitude of the lightcurve ranges from 0.14 mag (Rabinowitz et al. 2007) to 0.55 mag (Davies et al. 1998). This is perplexing, because the viewing geometry did not significantly change between the two sets of observations.

(9368) Esashi. Pray et al. (2008) derived unambiguous values of $P=2.9183 \pm 0.0002 \mathrm{~h}$ and $A=0.20 \mathrm{mag}$ for this asteroid. Ten SDSS observations were all of high quality. However, the 


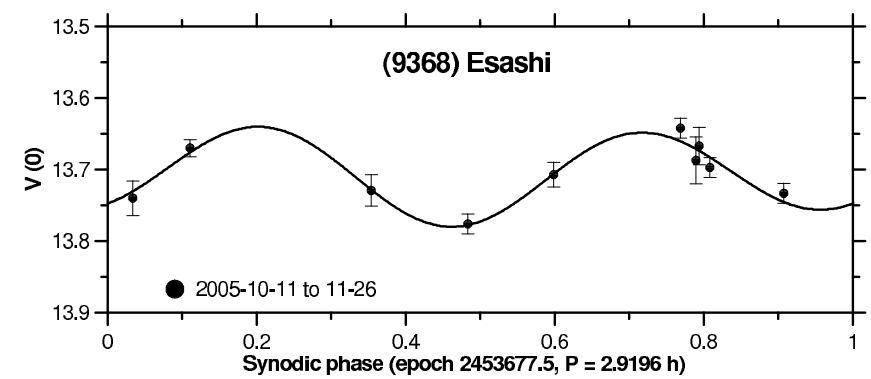

Fig. 8. Composite lightcurve of (9368) Esashi.

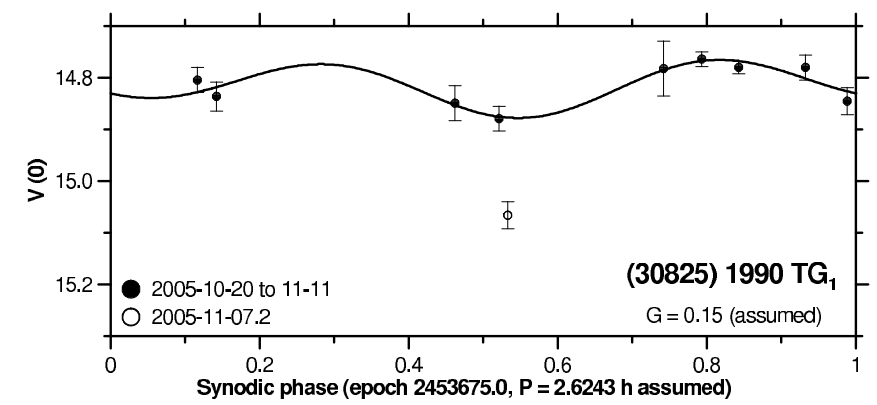

Fig. 9. Composite lightcurve of (30825) $1990 \mathrm{TG}_{1}$. One measurement denoted by an open circle was not considered in the Fourier fit. If the attenuation was real, it could be a sign of binarity.

period could not be determined unambiguously from the SDSS data due to the small amplitude of the lightcurve, the small number of observations, and the limitation of using only a secondorder Fourier fit. Of the many possible discrete periods that reasonably match the observations, the closest to that obtained by Pray is shown in Fig. 8. The exact solution is probably "overfitted" with a lower dispersion than the a priori estimated errors, so the $\mathrm{RMS}_{\mathrm{SDSS}}$ presented in Table 3 is underestimated, while the period uncertainty is actually larger than indicated by the fit dispersion. If the data were forced to the exact period found by Pray, the $\mathrm{RMS}_{\mathrm{SDSS}}$ with $n=2$ would be $0.030 \mathrm{mag}$, still within standard photometric accuracy.

(30825) $1990 \mathrm{TG}_{1}$. Higgins et al. (2006) worked this nearEarth asteroid in 2005 January and February and derived an unambiguous solution of $P=2.62428 \pm 0.00005 \mathrm{~h}$. The SDSS observations were made in 2005 October and November. Since the shape of the lightcurves may have changed between the times of the two data sets, the relative and SDSS data were not combined into a single set. The SDSS data for this asteroid were sparse. This made it difficult to find the period because of the small amplitude. One observation had to be excluded as an apparent outlier in $V$ even though it did not appear to be one based on color indices.

Potentially an asteroid with a rotation rate between 2 and $5 \mathrm{~h}$ and a low amplitude lightcurve could be a binary system, in which case eclipse/occultation events may be observed if the viewing geometry and orbit alignment are just right (Pravec et al. 2006). It is possible that the excluded data were the result of such mutual events; however, this cannot be confirmed based on the existing data (see Fig. 9). There are no reports of the asteroid being or suspected of being binary, even after radar measurements in 2005 by Benner et al. (2008). The RMS SDSS for the rest of the observations was within 0.05 mag for any period solution. Therefore the period derived by Higgins et al. (2006) was used here to display the scatter of the SDSS data.

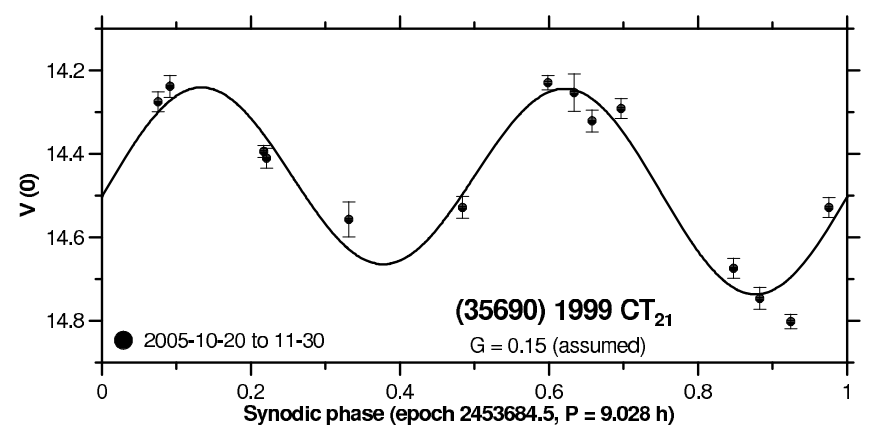

Fig. 10. Composite lightcurve of (35690) $1999 \mathrm{CT}_{21}$.

(35690) $1999 \mathrm{CT}_{21}$. The SDSS data for this asteroid led to several plausible periods with one of them (Fig. 10) very close to the one found by Pietrukowicz et al. (2006). Using two sessions from consecutive nights, they reported a reasonably secure result of $P=9.06 \pm 0.02 \mathrm{~h}$ with a large lightcurve amplitude of $A=0.60 \pm 0.02 \mathrm{mag}$. All 14 SDSS observations were used, because there were no apparent outliers in the $V$ band or in the color indices. However, a slightly larger scatter in color indices was observed, probably because the object was fainter than the asteroids discussed previously. Only a secondorder Fourier fit could be used, resulting in a relatively large residual of $\mathrm{RMS}_{\mathrm{SDSS}} \approx 0.06 \mathrm{mag}$. This could be reduced slightly (to $\approx 0.05 \mathrm{mag}$ ) by excluding some observations, increasing to a third-order fit, and setting $G$ closer to 0.2 . However, it was decided to present all data as they were using the default $G$ value. The derived value for $H$ using the SDSS data again differed significantly from the $H_{\mathrm{MPC}}$ value (14.47 and 14.0, respectively).

\section{Additional absolute magnitude estimates and seven probable lightcurves}

As shown above, it is possible to use reliable, calibrated SDSS observations to find the absolute magnitude $H$ for an asteroid when the rotation rate $P$ is known. Indeed, if part of the observations are made at small solar phase angles, and assuming the observations cover the entire lightcurve, it is possible to obtain reasonable $H$ values even without knowing $P$. Observations for all frequently observed asteroids listed in Table 1 were analyzed with the aim of finding values for $H$. For simplicity, the default of $G=0.15$ was assumed. After excluding apparent outliers on the basis of color indices such as $(B-V),(i-z)$, tentative synodic periods were searched using a low-order Fourier fit. Usually a number of solutions were found that matched the observations with relatively low root mean square residuals. Some of the derived periods (and amplitudes of the lightcurves) are listed in Table 4. Despite not knowing the actual periods, a very low scatter in $H$ values was obtained when using several tentative periods for each object in Table 1. One can compare them with the crude MPC values $H_{\text {MPC }}$ (the MPC also assumes $G=0.15$ ). It seems that 64 asteroids (from Tables 3 and 4) are usually fainter than expected, as the median value of $H-H_{\mathrm{MPC}}=0.26$, while its average value is 0.28 . Three asteroids with $G$ other than 0.15 have negligible influence on the results. This agrees with the result obtained by Jurić et al. (2002), who found the median value of $H-H_{\mathrm{MPC}} \approx 0.2$. Despite a large scatter in the data, a slight correlation between $H-H_{\mathrm{MPC}}$ and the amplitude of the lightcurve was found (Fig. 11). 
Table 4. Tentative results of the analysis for asteroids from Table 1.

\begin{tabular}{|c|c|c|c|c|c|}
\hline \multicolumn{2}{|c|}{ Asteroid } & \multirow{2}{*}{$\begin{array}{r}H_{\mathrm{MPC}} \\
10.8\end{array}$} & \multirow{2}{*}{$\begin{array}{l}H \\
11.51 / 11.51 / 11.52\end{array}$} & \multirow{2}{*}{$\begin{array}{l}P \\
\mathrm{~h} \\
(3.2895 / 5.0158 / 6.3792)\end{array}$} & \multirow{2}{*}{$\begin{array}{l}\begin{array}{l}A \\
\operatorname{mag}\end{array} \\
0.23 / 0.35 / 0.39\end{array}$} \\
\hline$(1947)$ & Iso-Heikkilä & & & & \\
\hline (8914) & Nickjames & 12.8 & $12.81 / 12.82 / 12.83$ & $(5.0669 / 5.347 / 6.406)$ & $0.20 / 0.20 / 0.15$ \\
\hline (12104) & Chesley & 12.4 & 12.76 & 3.9456 & 0.75 \\
\hline (32257) & $2000 \mathrm{OW}_{52}$ & 13.2 & $13.13(13.12 / 13.12)$ & $11.413(6.6990 / 21.785)$ & $0.28(0.26 / 0.26)$ \\
\hline (35774) & $1999 \mathrm{JL}_{9}$ & 13.9 & $14.27 / 14.25 / 14.25$ & $(2.1312 / 7.860 / 22.11)$ & $0.23 / 0.15 / 0.17$ \\
\hline (39132) & $2000 \mathrm{WU}_{58}$ & 13.5 & $14.32(14.33 / 14.30)$ & $20.663(14.439 / 36.31)$ & $0.69(0.60 / 0.62)$ \\
\hline (42306) & $2001 \mathrm{VU}_{46}$ & 14.5 & $14.86 / 14.86$ & $(2.2537 / 2.3651)$ & $0.20 / 0.20$ \\
\hline$(57252)$ & $2001 \mathrm{QS}_{96}$ & 13.0 & $13.60 / 13.61 / 13.60$ & $(7.209 / 10.310 / 11.427)$ & $0.36 / 0.41 / 0.30$ \\
\hline$(58673)$ & $1997 \mathrm{YA}_{9}$ & 14.2 & $14.87 / 14.87 / 14.94$ & $(6.918 / 9.731 / 19.365)$ & $0.47 / 0.52 / 0.71$ \\
\hline$(62781)$ & $2000 \mathrm{UE}_{23}$ & 14.9 & $14.84 / 14.80 / 14.85$ & $(17.95 / 28.94 / 71.7)$ & $0.8 / 1.1 / 1.1$ \\
\hline (63029) & $2000 \mathrm{WH}_{57}$ & 13.8 & $14.02 / 14.02 / 14.02$ & $(2.2667 / 8.693 / 13.641)$ & $0.25 / 0.19 / 0.19$ \\
\hline$(63376)$ & $2001 \mathrm{HA}_{38}$ & 16.5 & $16.10 / 16.09 / 16.09$ & $(2.0122 / 9.439 / 9.735)$ & $0.17 / 0.14 / 0.14$ \\
\hline$(65200)$ & $2002 \mathrm{CL}_{294}$ & 14.2 & $14.65 / 14.59$ & $(2.4206 / 6.461)$ & $0.48 / 0.38$ \\
\hline$(68480)$ & $2001 \mathrm{TQ}_{49}$ & 14.9 & $15.52 / 15.53 / 15.46$ & $(2.6982 / 4.4044 / 17.246)$ & $0.40 / 0.39 / 0.32$ \\
\hline (79530) & $1998 \mathrm{QD}_{4}$ & 15.8 & $16.02 / 16.03 / 16.04$ & $(2.9623 / 12.117 / 24.51)$ & $0.20 / 0.20 / 0.20$ \\
\hline (88005) & $2000 \mathrm{UR}_{44}$ & 14.1 & $14.22 / 14.22 / 14.23$ & $(11.726 / 15.524 / 22.958)$ & $0.26 / 0.27 / 0.26$ \\
\hline$(89891)$ & $2002 \mathrm{CQ}_{256}$ & 15.3 & $15.14 / 15.15$ & $(2.2512 / 8.258)$ & $0.34 / 0.31$ \\
\hline$(101812)$ & $1999 \mathrm{JD}_{7}$ & 15.1 & $15.46 / 15.46 / 15.48$ & $(2.47433 / 7.420 / 9.354)$ & $0.24 / 0.22 / 0.22$ \\
\hline (106590) & $2000 \mathrm{WQ}_{107}$ & 14.2 & $14.76 / 14.73$ & $(2.77757 / 21.67)$ & $(0.19 / 0.16)$ \\
\hline (110634) & $2001 \mathrm{TY}_{165}$ & 14.6 & $15.04 / 15.03$ & $(2.75308 / 4.9311)$ & $0.29 / 0.26$ \\
\hline (110755) & $2001 \mathrm{UT}_{13}$ & 14.9 & $15.35 / 15.38$ & $(46.9 / 49.0)$ & $0.8 / 0.75$ \\
\hline (119574) & $2001 \mathrm{VT}_{90}$ & 15.3 & $15.80 / 15.78$ & $(2.9245 / 43.40)$ & $0.24 / 0.24$ \\
\hline$(119651)$ & $2001 \mathrm{XE}_{49}$ & 14.8 & 15.28 & $(2.8458)$ & 0.39 \\
\hline$(124135)$ & $2001 \mathrm{LS}$ & 16.0 & $15.96 / 15.95$ & $(3.6217 / 5.4315)$ & $(0.17 / 0.22)$ \\
\hline$(125136)$ & $2001 \mathrm{UY}_{61}$ & 15.3 & $15.49 / 15.49$ & $(2.94565 / 11.596)$ & $0.23 / 0.14$ \\
\hline (125779) & $2001 \mathrm{XL}_{145}$ & 15.1 & $15.47 / 15.42 / 15.42$ & $(2.57199 / 7.655 / 11.251)$ & $0.29 / 0.28 / 0.28$ \\
\hline (128018) & $2003 \mathrm{JA}_{14}$ & 14.3 & $14.42 / 14.44 / 14.44$ & $(4.8539 / 8.083 / 12.192)$ & $0.34 / 0.36 / 0.31$ \\
\hline (128343) & $2004 \mathrm{GQ}_{5}$ & 16.1 & $16.20 / 16.19 / 16.23$ & $(2.4776 / 4.2251 / 26.53)$ & $0.25 / 0.19 / 0.3$ \\
\hline (148844) & $2001 \mathrm{VA}_{22}$ & 14.9 & 15.40 & $(3.1961)$ & $(0.5)$ \\
\hline (149182) & $2002 \mathrm{JX}_{69}$ & 14.5 & $14.98 / 14.98$ & $(2.8950 / 3.2926)$ & $0.30 / 0.30$ \\
\hline$(151057)$ & $2001 \mathrm{VO}_{14}$ & 15.6 & $15.86 / 15.86$ & $20.631 / 36.20$ & $0.6 / 0.6$ \\
\hline$(153695)$ & $2001 \mathrm{US}_{35}$ & 15.6 & $15.84 / 15.86 / 15.86$ & $(4.0108 / 18.61 / 30.43)$ & $0.39 / 0.34 / 0.33$ \\
\hline (153822) & $2001 \mathrm{WH}_{37}$ & 15.8 & $16.05 / 16.04$ & $(5.6388 / 7.373)$ & $0.18 / 0.20$ \\
\hline (153879) & $2001 X_{141}$ & 16.2 & $16.49 / 16.52$ & $(2.3455 / 4.6939)$ & $0.13 / 0.18$ \\
\hline (153937) & $2001 \mathrm{YG}_{97}$ & 15.2 & $15.63 / 15.65$ & $(3.6951 / 16.313)$ & $0.18 / 0.22$ \\
\hline$(156751)$ & $2002 \mathrm{XL}_{92}$ & 16.8 & $17.28(17.28)$ & $6.5287(5.7470)$ & $0.8(0.9)$ \\
\hline (160318) & $2003 \mathrm{JR}_{8}$ & 14.8 & $14.98 / 14.99$ & $(4.0945 / 21.83)$ & $0.28 / 0.24$ \\
\hline (214479) & $2005 \mathrm{~TB}_{73}$ & 14.4 & $14.75 / 14.75$ & $20.594 / 36.06$ & $0.84 / 0.96$ \\
\hline (215187) & $2000 \mathrm{LQ}_{25}$ & 15.5 & $16.06 / 16.05$ & $17.735 / 12.945$ & $0.61 / 0.58$ \\
\hline (219686) & $2001 \mathrm{WE}_{37}$ & 17.0 & $17.15(17.15 / 17.15)$ & $10.214(8.420 / 12.979)$ & $0.49(0.45 / 0.40)$ \\
\hline (221267) & 2005 US $_{313}$ & 15.7 & $16.00 / 16.03 / 15.99$ & $(3.8397 / 6.8759 / 14.514)$ & $0.10 / 0.19 / 0.12$ \\
\hline \multirow[t]{13}{*}{$(225873)$} & $2001 \mathrm{YL}_{4}$ & 15.8 & $16.13 / 16.11 / 16.09$ & $(2.8464 / 3.1010 / 7.2122)$ & $0.39 / 0.39 / 0.42$ \\
\hline & $1992 \mathrm{WW}_{6}$ & 16.2 & 16.45 & 6.3666 & 0.77 \\
\hline & $2003 \mathrm{GG}_{51}$ & 15.4 & $15.45 / 15.45 / 15.47$ & $(5.9437 / 6.785 / 8.423)$ & $0.45 / 0.49 / 0.50$ \\
\hline & $2005 \mathrm{RF}_{47}$ & 17.4 & $17.39 / 17.42$ & $13.556 / 18.887$ & $0.55 / 0.67$ \\
\hline & $2005 \mathrm{SF}_{286}$ & 17.4 & $17.38 / 17.33 / 17.34$ & $(2.1145 / 2.36990 / 8.889)$ & $0.40 / 0.59 / 0.40$ \\
\hline & $2005 \mathrm{SS}_{287}$ & 16.4 & $16.46 / 16.50 / 16.52$ & $(11.395 / 14.791 / 21.40)$ & $0.59 / 0.59 / 0.62$ \\
\hline & $2005 \mathrm{UV}_{108}$ & 15.5 & $15.73 / 15.75 / 15.76$ & $(4.1676 / 11.634 / 22.62)$ & $0.53 / 0.48 / 0.47$ \\
\hline & $2005 \mathrm{UC}_{130}$ & 16.7 & $16.85 / 16.84 / 16.85$ & $(4.4018 / 6.4083 / 21.83)$ & $0.18 / 0.20 / 0.20$ \\
\hline & $2005 \mathrm{UO}_{213}$ & 15.7 & $15.78 / 15.76$ & $(2.7128 / 30.73)$ & $0.28 / 0.31$ \\
\hline & $2005 \mathrm{UU}_{396}$ & 15.2 & $15.49 / 15.49 / 15.50$ & $(5.812 / 7.689 / 11.288)$ & $0.25 / 0.26 / 0.26$ \\
\hline & $2005 \mathrm{VR}_{131}$ & 16.2 & $16.27 / 16.30 / 16.26$ & $(4.5051 / 5.2737 / 5.5483)$ & $0.26 / 0.36 / 0.29$ \\
\hline & $2005 \mathrm{VS}_{131}$ & 16.0 & $16.24 / 16.26 / 16.25$ & $(2.7107 / 6.246 / 15.67)$ & $0.33 / 0.29 / 0.26$ \\
\hline & $2007 \mathrm{EP}_{39}$ & 17.4 & 17.69 & 5.7700 & 0.51 \\
\hline
\end{tabular}

Data for several asteroids, for example (151057) $2001 \mathrm{VO}_{14}$, (214479) $2005 \mathrm{~TB}_{73}$, (215187) $2000 \mathrm{LQ}_{25}$, and $2005 \mathrm{RF}_{47}$, were in a state that only two periods were preferred. There were even some targets for which only one period dominated, and the $\mathrm{RMS}_{\text {SDSS }}$ for other periods was much larger. These asteroids are analyzed in more detail below, because it seemed that more reliable synodic periods might be found. The result can be verified by photometric observations in the future, at least for the brightest asteroids.
(12104) Chesley. The available SDSS observations of (12104) Chesley provided a nearly secure synodic period of $P=3.9456 \pm$ $0.0001 \mathrm{~h}$ (Fig. 12), mostly because of the large amplitude of the lightcurve: $A \approx 0.75$ mag (Fig. 13). The maximum synodicsidereal period difference for the period covered by observations is $\Delta P<0.0001 \mathrm{~h}$. When all the data were fitted with a second-order Fourier series, $\mathrm{RMS}_{\mathrm{SDSS}}=0.053 \mathrm{mag}$ and remained so in the range $0.14<G<0.18$. Outside this range, the $\mathrm{RMS}_{\mathrm{SDSS}}$ was larger. Thus, the best formal result is $G=0.16$ 


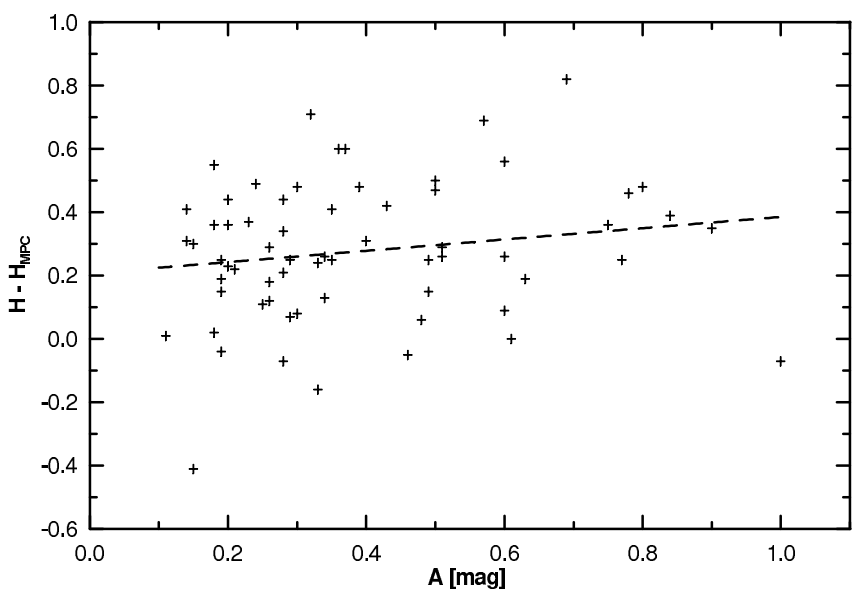

Fig. 11. Correlation between $H-H_{\mathrm{MPC}}$ and amplitude $A$ of the lightcurve.

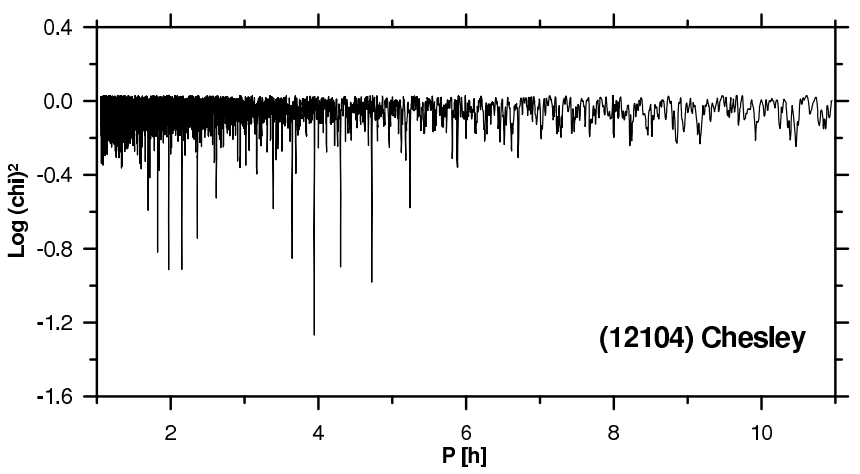

Fig. 12. Plot of the sum of square residuals vs. period for (12104) Chesley. The best bimodal lightcurve that matches the observations is found at $3.9456 \mathrm{~h}$. The other bimodal lightcurves are found at $3.6457 \mathrm{~h}$, $4.2995 \mathrm{~h}$ and $4.7228 \mathrm{~h}$.

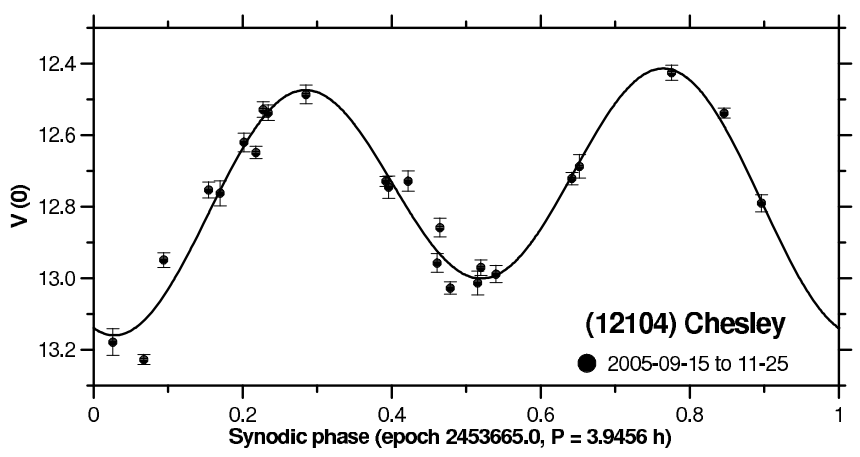

Fig. 13. Probable composite lightcurve of (12104) Chesley.

and $H=12.77$. Neither using higher-order Fourier series nor rejecting dubious observations based on color indices improved the fit. The RMS $S_{\text {SDSS }}$ for all other periods, including those with bimodal lightcurves, exceeded $0.07 \mathrm{mag}$. Even so, all periods led to the same $H$ for a given $G$ value.

(32257) $2000 \mathrm{OW}_{52}$. Despite a relatively low amplitude lightcurve of $0.28 \mathrm{mag}$, the SDSS observations of (32257) 2000 $\mathrm{OW}_{52}$ led to a reasonably secure result of $P=11.413 \pm 0.001 \mathrm{~h}$ (Figs. 14, 15). The maximum synodic-sidereal period difference was $\Delta P<0.001 \mathrm{~h}$. Unfortunately, there are usually many alias periods possible when only a sparse data set is available for an asteroid with a small amplitude lightcurve. Only one outlying

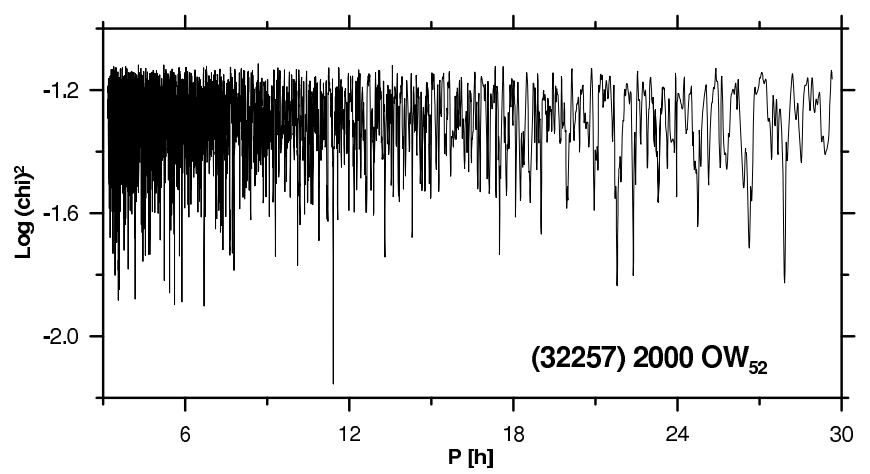

Fig. 14. Plot of the sum of square residuals vs. period for (32257) 2000 $\mathrm{OW}_{52}$. The third Fourier order was chosen. The best solutions would remain at the same positions with the fourth order.

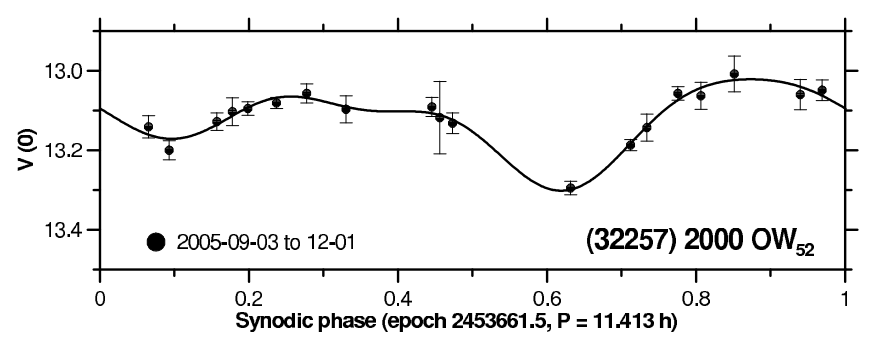

Fig. 15. Probable composite lightcurve of (32257) $2000 \mathrm{OW}_{52}$.

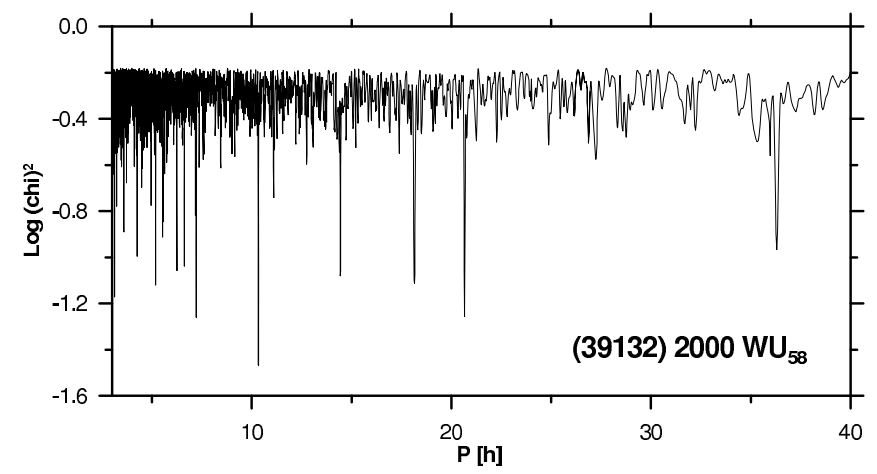

Fig. 16. Plot of the sum of square residuals vs. period for (39132) 2000 $\mathrm{WU}_{58}$. The bimodal lightcurves that match the observations are just at periods of $14.439 \mathrm{~h}, 20.663 \mathrm{~h}$ and $36.31 \mathrm{~h}$.

observation was excluded from the 2005 apparition data. The rest of the observations were fitted with a fourth-order Fourier series, which gave $\mathrm{RMS}_{\mathrm{SDSS}}=0.019 \mathrm{mag}$. The best formal result was $G=0.17\left(\mathrm{RM}_{\mathrm{SDSS}}=0.018 \mathrm{mag}\right)$ and $H=13.14$. The $\mathrm{RMS}_{\mathrm{SDSS}}$ for two other possible periods in Table 4 was $\approx 0.03 \mathrm{mag}$, while it was much higher for other values of $P$.

(39132) $2000 \mathrm{WU}_{58}$. The SDSS observations of (39132) $2000 \mathrm{WU}_{58}$ led to a nearly secure result of $P=20.663 \pm 0.004 \mathrm{~h}$ (Fig. 16), again thanks to the large amplitude of the lightcurve (Fig. 17). The maximum synodic-sidereal period difference was $\Delta P=0.004 \mathrm{~h}$. Four observations were excluded. The rest of observations were fitted with a fourth-order Fourier series leading to $\mathrm{RMS}_{\mathrm{SDSS}}=0.040 \mathrm{mag}$. Formally, the best result appeared to be for $G=0.01$ with $\mathrm{RMS}_{\mathrm{SDSS}}=0.032 \mathrm{mag}$, $P=20.664 \pm 0.003 \mathrm{~h}, A=0.73 \mathrm{mag}$ and $H=14.19$. No other solution with $\mathrm{RMS}_{\mathrm{SDSS}}<0.06$ mag was found. 


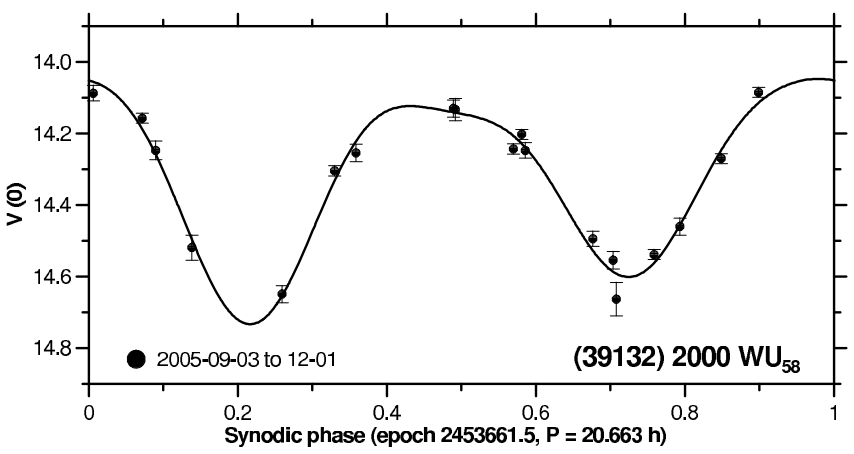

Fig. 17. Probable composite lightcurve of (39132) $2000 \mathrm{WU}_{58}$.

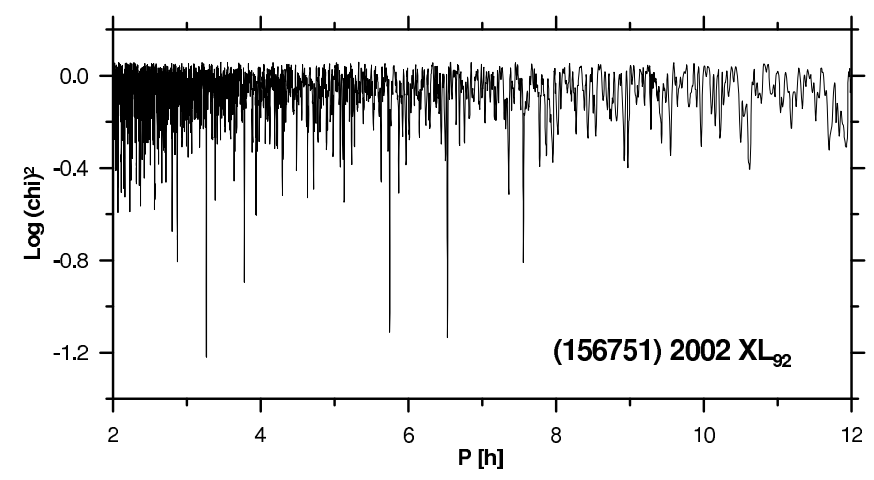

Fig. 18. Plot of the sum of square residuals vs. period for (156751) 2002 $\mathrm{XL}_{92}$. The bimodal lightcurves that match the observations are found between $5.7 \mathrm{~h}$ and $7.6 \mathrm{~h}$.

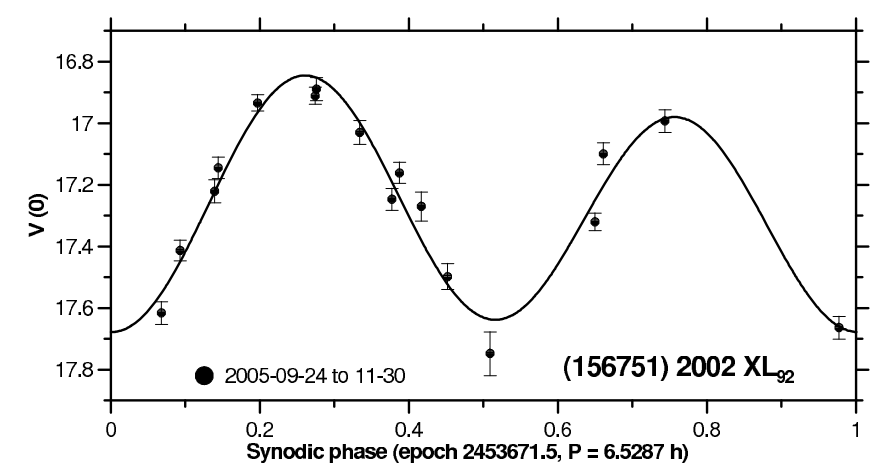

Fig. 19. Probable composite lightcurve of (156751) $2002 \mathrm{XL}_{92}$.

(156751) $2002 X_{92}$. Even though five SDSS observations were excluded, a nearly secure result of $P=6.5287 \pm 0.0007 \mathrm{~h}$ was obtained for (156751) $2002 \mathrm{XL}_{92}$ (Fig. 18). The maximum synodic-sidereal period difference was $\Delta P=0.0004 \mathrm{~h}$. Another solution of $5.7470 \mathrm{~h}$, still assuming a bimodal lightcurve, was less probable based on visual inspection. Both results led to $H=17.28$ with $G=0.15$. The amplitude was $A \approx 0.8-0.9$ mag (Fig. 19). With the data fitted to a second-order Fourier series, $\mathrm{RMS}_{\mathrm{SDSS}}=0.074 \mathrm{mag}$. Higher orders were not reliable.

Formally, the best result in terms of $\mathrm{RMS}_{\mathrm{SDSS}}$ was for the very low value of $G=-0.05$, which led to $H=17.11, P=$ $6.5289 \pm 0.0005 \mathrm{~h}, A=0.95 \mathrm{mag}$ and $\mathrm{RMS}_{\mathrm{SDSS}}=0.060 \mathrm{mag}$. The actual accuracy is unknown since the lightcurve can differ from the fit, but faint objects are usually of lower quality. Also, the true values of $H, G$, and $P$ may be slightly different due to missing high-quality observations in some parts of the composite lightcurve. For example, by including the 5 observations of lower reliability, the best result is $G=0.01, H=17.13$,

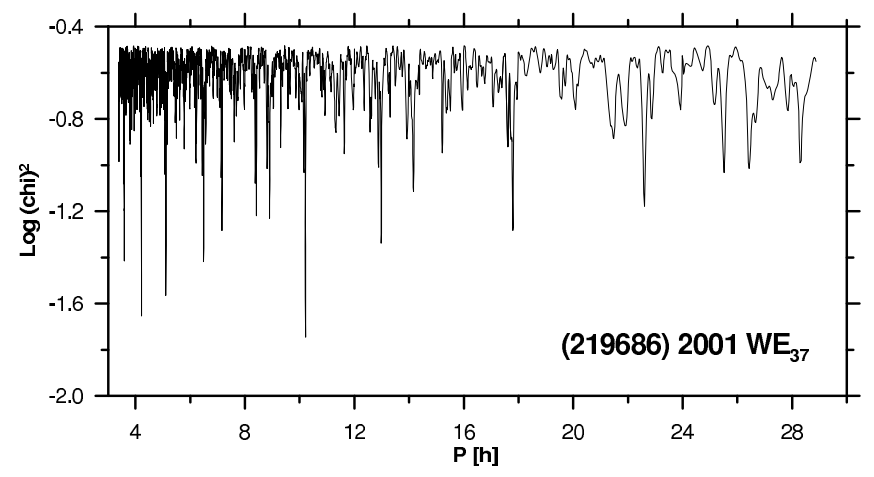

Fig. 20. Plot of the sum of square residuals vs. period for $2001 \mathrm{WE}_{37}$. The bimodal lightcurves that match the observations are found for periods $>7 \mathrm{~h}$, while the one at $8.9 \mathrm{~h}$ is still monomodal.

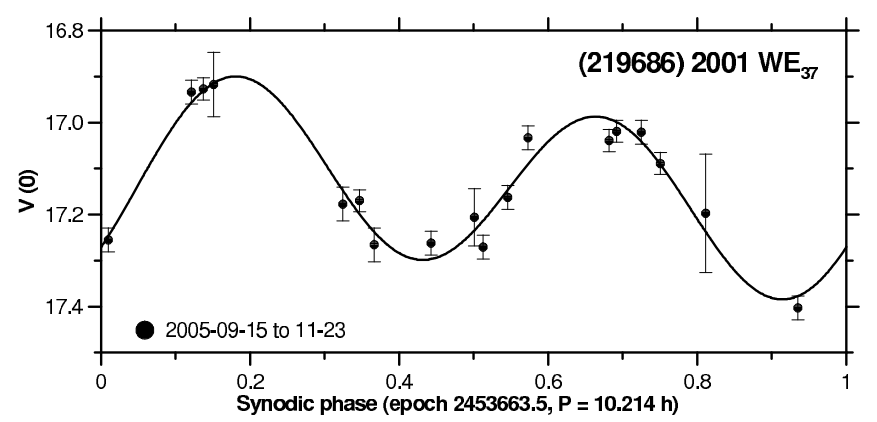

Fig. 21. Probable composite lightcurve of $2001 \mathrm{WE}_{37}$.

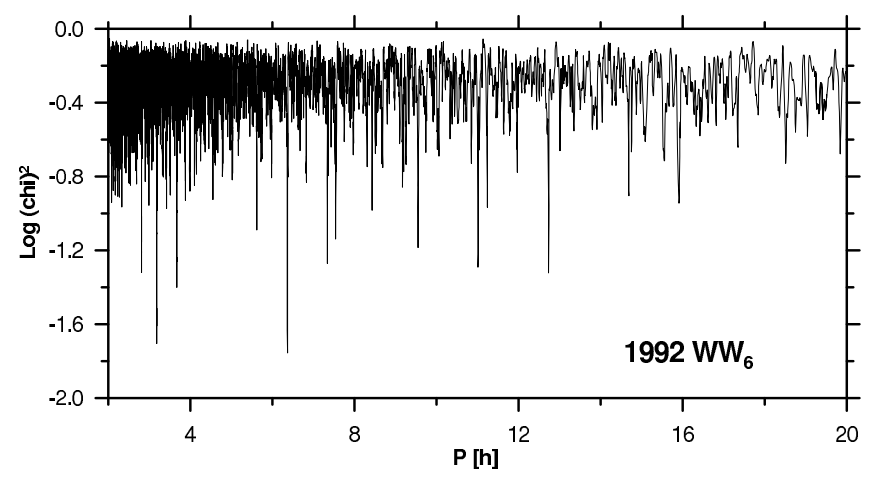

Fig. 22. Plot of the sum of square residuals vs. period for $1996 \mathrm{WW}_{6}$. The bimodal lightcurves that match the observations are found only between $5.6 \mathrm{~h}$ and $7.3 \mathrm{~h}$.

$P=6.5289 \pm 0.0006 \mathrm{~h}, A \approx 1.0 \mathrm{mag}$, and $\mathrm{RMS}_{\mathrm{SDSS}} \approx 0.13 \mathrm{mag}$ when using a second-order Fourier fit.

(219686) $2001 \mathrm{WE}_{37}$. There were four outliers (based on color indices) for this asteroid, but only two of them were rejected. The remaining two had no substantial influence on the results. Assuming the typical bimodal shape for a lightcurve, only a few candidate periods were found to match the SDSS observations well (Fig. 20). The best result, with $P=10.214 \pm 0.001 \mathrm{~h}$, $\Delta P=0.002 \mathrm{~h}, A=0.49 \mathrm{mag}$, is shown in Fig. 21. A secondorder Fourier fit gave the lowest $\mathrm{RMS}_{\mathrm{SDSS}}(0.036 \mathrm{mag})$ for $G=0.15$. Two other discrete periods are included in Table 4; the $\mathrm{RMS}_{\mathrm{SDSS}} \approx 0.06 \mathrm{mag}$ for each of those.

$1992 W_{6}$. After three observations were rejected, the SDSS data led to a unique period of $P=6.3666 \pm 0.0002 \mathrm{~h}$ (Fig. 22). 


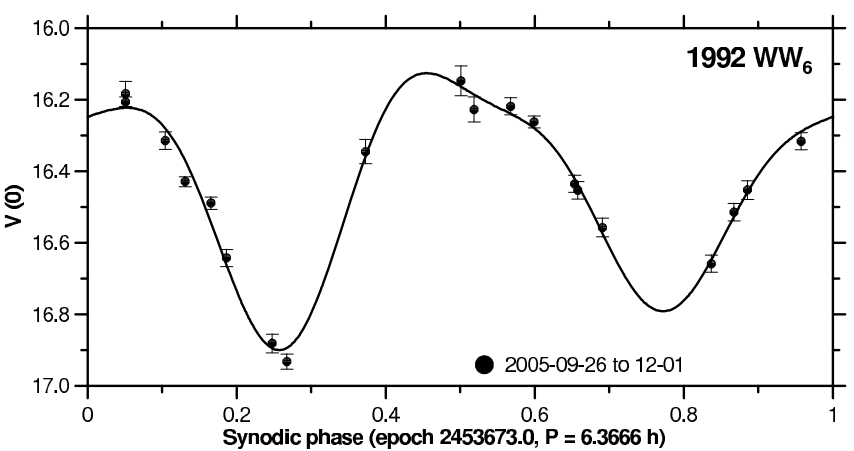

Fig. 23. Probable composite lightcurve of $1996 \mathrm{WW}_{6}$.

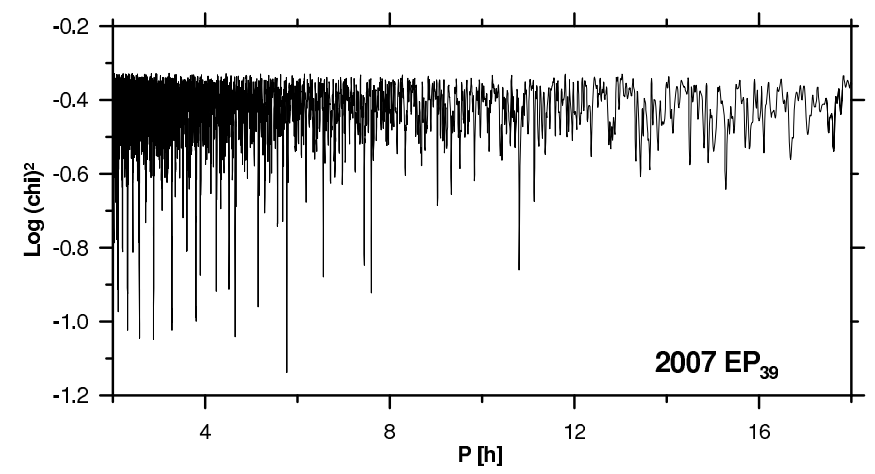

Fig. 24. Plot of the sum of square residuals vs. period for $2007 \mathrm{EP}_{39}$. The first bimodal lightcurve that matches the observations is found at $\approx 3.9 \mathrm{~h}$.

In fact, one of the rejected outliers had a large formal error, but even when included in the analysis the results did not change. The maximum synodic-sidereal period difference was $\Delta P=$ $0.0005 \mathrm{~h}$. A fourth-order Fourier fit gave $\mathrm{RMS}_{\mathrm{SDSS}}=0.038 \mathrm{mag}$ (Fig. 23). A fifth-order fit decreased the $\mathrm{RMS}_{\mathrm{SDSS}}$ value further, but this resulted in a somewhat strange composite lightcurve. Formally, the lowest $\mathrm{RMS}_{\mathrm{SDSS}}=0.026 \mathrm{mag}$ was for $G=0.38$, while $P=6.3660 \pm 0.0002 \mathrm{~h}, A=0.75 \mathrm{mag}$, and $H=16.63$.

$2007 \mathrm{EP}_{39}$. At the time of the SDSS observations, this very faint object $(V>20)$ was unknown. Four observations were outliers in some color indices. Due to the indication of a large amplitude $(A=0.51 \mathrm{mag})$, the period search was confined to bimodal lightcurves. This led to a few probable results (Fig. 24), the best one of which is shown in Fig. 25. It is based on a secondorder Fourier fit with $P=5.7700 \pm 0.0005 \mathrm{~h}, \Delta P=0.0004 \mathrm{~h}$, and $\mathrm{RMS}_{\text {SDSS }}=0.065 \mathrm{mag}$. Two outliers were also included because they did not seem to influence the results. One has a large error in one color index, while the other is located at an uncovered part of the composite lightcurve. Formally, $G=0.25$, $P=5.7698 \pm 0.0005 \mathrm{~h}$, and $H=17.78$ gave the lowest value for the $\mathrm{RMS}_{\text {SDSS }}(0.059 \mathrm{mag})$.

\section{Conclusions}

As expected, calibrated SDSS data alone are not sufficient for finding secure rotation periods of asteroids, mostly due to a lack of observations for any given asteroid. The only exception is for asteroids with large lightcurve amplitudes observed multiple times. However, the SDSS observations can be used to refine rotation periods, especially if some crude limits are known from

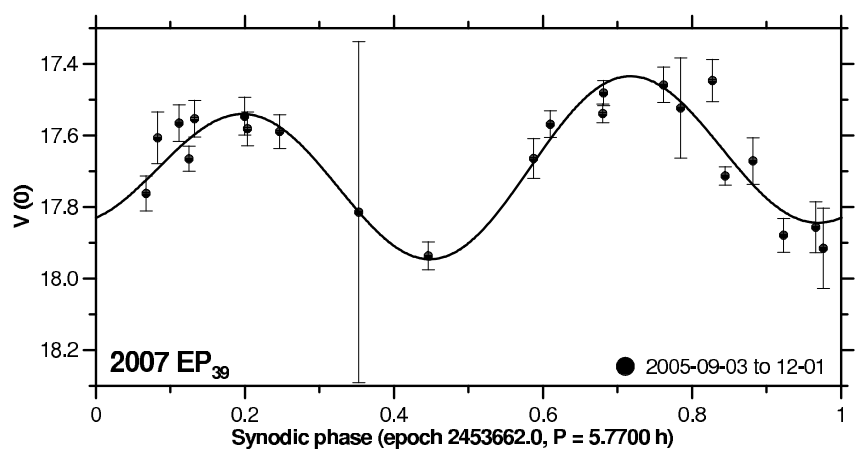

Fig. 25. Probable composite lightcurve of $2007 \mathrm{EP}_{39}$.

other photometric observations and, again, preferably for larger amplitude lightcurves with simpler shapes. Similarly, absolute magnitudes can be derived precisely for asteroids with a sufficient number of observations. The asteroids studied in this paper were found to be mostly fainter than predicted from the absolute magnitude values given by the Minor Planet Center in its Orbit Database.

The Sloan Digital Sky Survey observations appear to be standard in terms of photometric quality. However, special attention should be paid to the reliability of individual observations. After removing all outlying observations based on color indices (which was not always easy to do), the actual error was found to be about 0.03 mag on average for bright targets $(V<18)$.

With proper care, it is possible for asteroid photometrists to use the SDSS data to find rotation rates and compare those results against those obtained with dense data sets.

Acknowledgements. I am grateful to Petr Pravec, Ondřejov Observatory, Czech Republic, for his frequent advice and his ALC software used in the data analysis. Thanks also go to Donald P. Pray, Carbuncle Hill Observatory, Greene, Rhode Island, USA, and Marek Husárik, Skalnaté Pleso Observatory, Slovakia, for providing their published data to reanalyze by ALC software, but also to Brian D. Warner, Palmer Divide Observatory, Colorado Springs, USA, for his kind corrections to improve the readability of the text and Alan W. Harris, Space Science Institute, La Canada, USA, for his kind additional comments. I also appreciate final referee comments by Tomasz Kwiatkowski. The work at Modra has been supported by the Slovak Grant Agency for Science VEGA, Grant 2/0016/09. The work at Ondřejov has been supported by the Grant Agency of the Czech Republic, Grant 205/09/1107.

Funding for the creation and distribution of the SDSS Archive has been provided by the Alfred P. Sloan Foundation, the Participating Institutions, the National Aeronautics and Space Administration, the National Science Foundation, the US Department of Energy, the Japanese Monbukagakusho, and the Max Planck Society. The SDSS Web site is http://wWw. sdss.org/.

The SDSS is managed by the Astrophysical Research Consortium (ARC) for the Participating Institutions. The Participating Institutions are The University of Chicago, Fermilab, the Institute for Advanced Study, the Japan Participation Group, The Johns Hopkins University, the Korean Scientist Group, Los Alamos National Laboratory, the Max-Planck-Institute for Astronomy (MPIA), the Max-Planck-Institute for Astrophysics (MPA), New Mexico State University, University of Pittsburgh, University of Portsmouth, Princeton University, the United States Naval Observatory, and the University of Washington.

\section{References}

Benner, L. A. M., Ostro, S. J., Magri, C., et al. 2006, Icarus, 198, 294

Brown, W. R., \& Luu, J. X. 1997, Icarus, 126, 218

Brucker, M., Romanishin, W. J., Tegler, S. C., Consolmagno, G. J., \& Grundy, W. M. 2008, BAAS, 40, 483 
Davies, J. K., McBride, N., Ellison, S. L., Green, S. F., \& Ballantyne, D. R. 1998, Icarus, 134, 213

Fukugita, M., Ichikawa, T., Gunn, J. E., et al. 1996, AJ, 111, 1748

Harris, A. W., Young, J. W., Bowell, E., et al. 1989, Icarus, 77, 171

Higgins, D., Pravec, P., Kusnirak, P., et al. 2006, Minor Planet Bull., 33, 8

Ivezić, Ž., Jurić, M., Lupton, R. H., Tabachnik, S., \& Quinn, T. 2002, in Survey and Other Telescope Technologies and Discoveries, ed. J. A. Tyson, \& S. Wolff, Proc. SPIE, 4836, 98

Jurić, M., Ivezić, Ž., Lupton, R. H., et al. 2002, AJ, 124, 1776

Jurić, M., Ivezić, Ž., Lupton, R., \& SDSS Collaboration 2007, BAAS, 39, 828

Kern, S. D., McCarthy, D. W., Buie, M. W., et al. 2000, ApJ, 542, L155

Pietrukowicz, P., Michalowski, T., Pych, W., Kaluzny, J., \& Thompson, I. B. 2006, Minor Planet Bull., 33, 80
Pravec, P., Harris, A. W., Scheirich, P., et al. 2005, Icarus, 173, 108 Pravec, P., Scheirich, P., Kušnirák, P., et al. 2006, Icarus, 181, 63 Pray, D. P. 2006, Minor Planet Bull., 33, 4

Pray, D. P., Galád, A., Gajdoš, Š., et al. 2006, Minor Planet Bull., 33, 92 Pray, D. P., Galád, A., Husárik, M., et al. 2008, Minor Planet Bull., 35, 34 Rabinowitz, D. L., Schaefer, B. E., \& Tourtellotte, S. W. 2007, AJ, 133, 26 Sauppe, J., Torno, S., Lemke-Oliver, R., et al. 2007, Minor Planet Bull., 34, 119

Stephens, R. D. 2003, Minor Planet Bull., 30, 31

Warner, B. D., Harris, A. W., \& Pravec, P. 2009, Icarus, 202, 134

Wisniewski, W. Z., Michałowski, T. M., Harris, A. W., et al. 1997, Icarus, 126, 395

York, D. G., Adelman, J., Anderson Jr., J. E., et al. 2000, AJ, 120, 1579 